\title{
Recognized homonymous hemianopsia and delirium during the admission examination leading to diagnosis and appropriate treatment of a new stroke
}

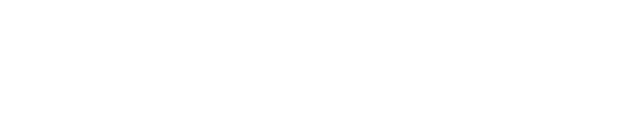

\section{Konstantin S Tsymbalov Douglas R Fetkenhour}

Department of Physical Medicine and Rehabilitation, University of Rochester Medical Center, Rochester, NY, USA
Correspondence: Konstantin S Tsymbalov Department of Physical Medicine and Rehabilitation, University of Rochester Medical Center, 60I Elmwood Avenue, Box 664, Rochester, NY 15642, USA Email ktsymbalov@yahoo.com

\begin{abstract}
This case report describes the detection of homonymous hemianopsia and delirium during the admission physical examination of a patient with esophageal adenocarcinoma, resulting in the new diagnosis of subacute hemorrhagic stroke. The poststroke visual field defect can result in significant disability and reduction in quality of life. Patients with visual field cut show a severely reduced quality of life and require additional neuropsychological and visual rehabilitation. Only thorough physical examination is able to challenge prior negative positron emission tomography scan, leading to the diagnosis of subacute stroke and, following appropriate treatment, secondary stroke prophylaxis and rehabilitation, instead of brain radiation and chemotherapy.
\end{abstract}

Keywords: physical examination, homonymous hemianopsia, stroke, esophageal tumor

\section{Case presentation}

The verbal informed patient consent was acquired for the case report publication during the acute rehabilitation. Institutional review board approval was not sought for this case report. Whether a patient visits a clinic or is admitted to the hospital, the importance of a detailed physical examination must be emphasized as a resource leading to an appropriate diagnosis and treatment. Every medical student starts his or her medical career with learning how to take a good history from the patient and perform a thorough physical examination. This report details such a case where a thorough initial physical examination resulted in the diagnosis of subacute hemorrhagic stroke in a patient with esophageal adenocarcinoma.

A 65-year-old male, an experienced civil engineer, with a history of gastroesophageal reflux disease and hiatal hernia with a new complaint of progressive difficulty swallowing associated with a recent 20-pound weight loss was referred to an oncologist. The patient was evaluated by the oncology specialist 2 weeks later, and esophagogastroduodenoscopy was performed that revealed a distal esophageal mass, which was identified through a biopsy as moderately differentiated adenocarcinoma. Subsequent positron emission tomography and computed tomography (CT) scan studies performed 2 weeks later revealed distal esophageal wall thickening with an increased uptake extending into the proximal stomach but no brain lesions. The patient received neoadjuvant chemotherapy and en bloc esophagectomy with cervical esophagostomy, pyloroplasty, and jejunostomy feeding tube placement during the subsequent admission to a tertiary medical center. The patient was medically stabilized and evaluated for admission to a rehabilitation unit. 
During the rehabilitation admission examination, the patient was found to have left homonymous hemianopsia as a new physical examination finding. The patient also had some confusion with decreased initiation, not being able to perform at his educational level. Considering these new findings along with his recent history of invasive and possibly metastatic esophageal adenocarcinoma, the admission team decided to proceed with a magnetic resonance imaging (MRI) study of the brain. The differential diagnosis of brain metastasis versus a new stroke was proposed by the primary treating team.

The MRI of the brain revealed a new brain mass within the right parietal-occipital lobe, measuring $5.2 \times 3.8 \times 5 \mathrm{~cm}$ (Figure 1A and B). According to the radiology department report, it was likely a primary brain neoplasm, such as a neural or glial tumor, or lymphoma, but less likely a focus of metastatic disease, given its appearance. However, according to the oncology department evaluation, the lesion was more consistent with a metastatic tumor than a primary brain lesion.

The patient started to have seizure activity and delirium during the rehabilitation course. He was treated with oral antiepileptics. At the same time, the patient complained of having shortness of breath and was diagnosed as having pleural effusions. A pathological examination of thorasentesis concluded that no malignant cells were present in the pleural effusion fluid. There was also a concern regarding minimal intracerebral hemorrhage presented on the MRI scan. A series of two head CT scans within 24-48 hours were performed to rule out any acute hemorrhage (Figure 2A).

The tumor was found to be accessible for resection per neurosurgery evaluation with a plan for tumor resection when the patient was medically stable. A subsequent craniotomy and lesion biopsy followed by pathology investigation surprisingly identified the lesion as a subacute cortical ischemic stroke negative for neoplasm. A postoperative head CT scan showed no acute hemorrhage (Figure 2B). The patient did well postoperatively. He was provided with appropriate treatment and secondary stroke prophylaxis and was able to complete the rehabilitation on the acute rehabilitation floor.

\section{Discussion}

In the case presented, the diagnosis of subacute ischemic stroke was made on the basis of focal findings during a detailed admission physical examination in the universitybased acute rehabilitation unit. The patient was found to have mild chronic delirium with an inability to concentrate, decreased initiation, affected short-term memory, and homonymous hemianopsia. All new focal deficit findings, such as homonymous hemianopsia, require additional attention and further investigation.

There are several case reports in the literature strongly suggesting that physicians have to conduct a complete
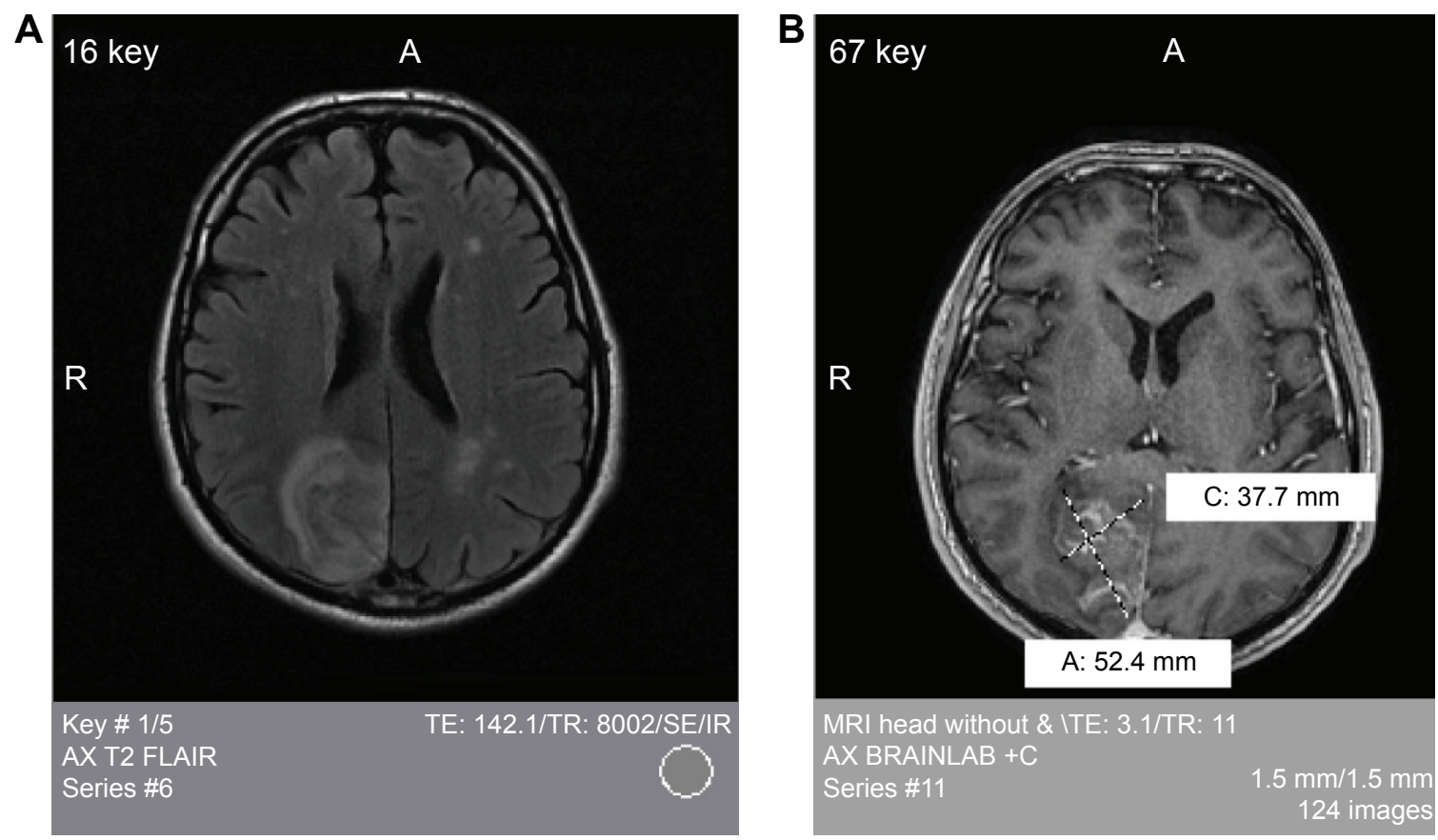

Figure I Magnetic resonance imaging (MRI) of the head without contrast.

Notes: (A) An axial magnetic resonance imaging scan demonstrating an enhanced mass within the right parietal-occipital lobe and artifacts. (B) An axial magnetic resonance imaging scan demonstrating an enhanced mass within the right parietal-occipital lobe measuring $5.2 \times 3.8 \times 5 \mathrm{~cm}$. 

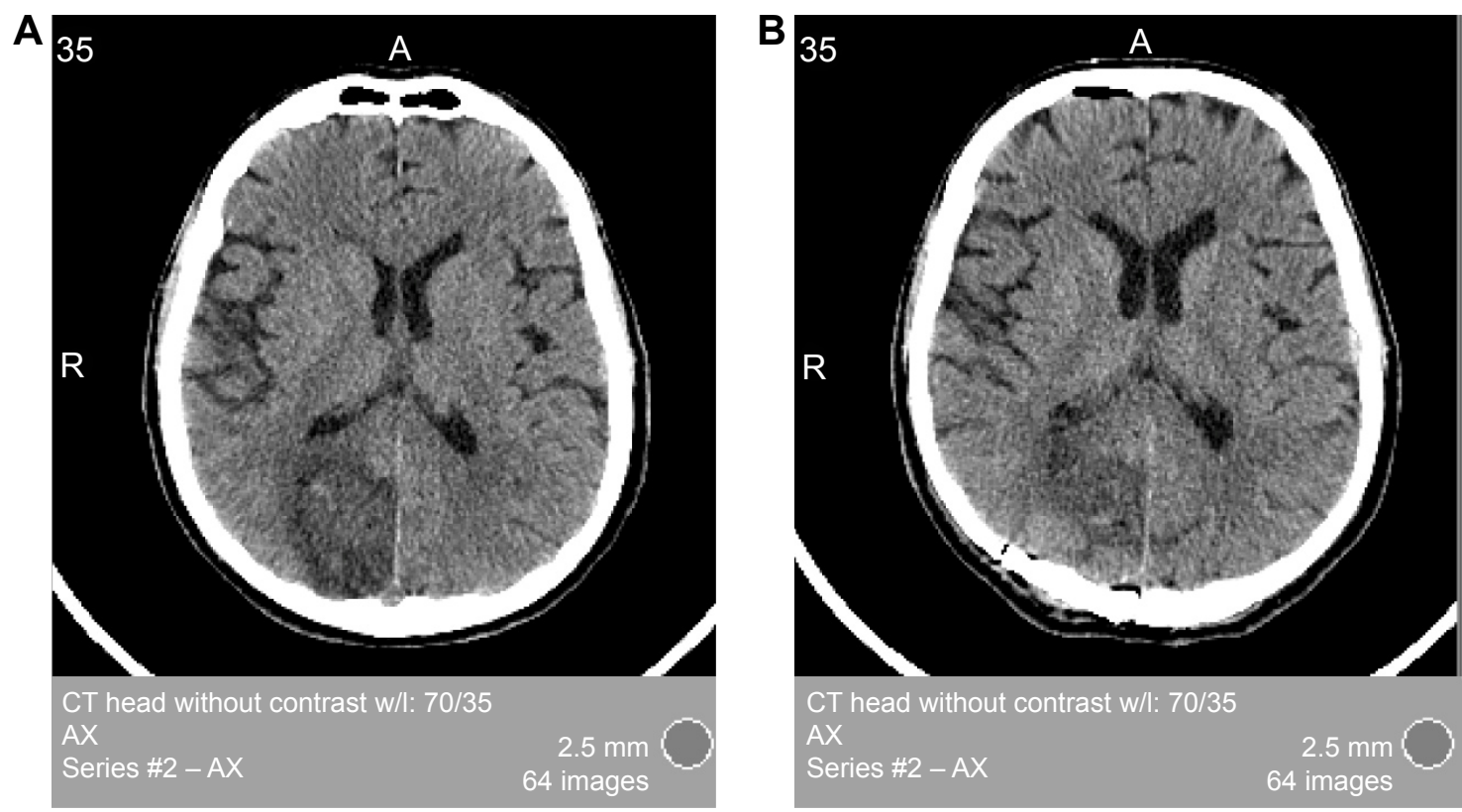

Figure 2 Computed tomography (CT) scan of the head without contrast.

Notes: (A) An axial CT imaging scan demonstrating a mass in the right parietal-occipital lobe with a mass effect on the occipital horn of the right lateral ventricle and surrounding edema. (B) An axial CT imaging scan demonstrating postoperative changes following right occipital craniotomy and biopsy, including a small focus of hemorrhage likely at the biopsy site.

Abbreviation: $\mathrm{CT}$, computed tomography.

medical history and arrange a specialist consultation for all patients' focal findings such as visual field cuts. ${ }^{1}$ The top differential diagnostic considerations for a presentation of homonymous hemianopsia for this patient were metastatic lesion from a known esophageal carcinoma, stroke, and a primary brain tumor. According to the MRI report, the lesion was "likely primary brain neoplasm, such as a neural or glial tumor, or lymphoma" and "less likely a focus of metastatic disease given the appearance" on the scan images (Figure 1A and B). It is very important to use the knowledge of the morphological presentation, and probability for each type of malignancy, to perform the second step in the evaluation of the patient when the clinical presentation led to the discovery of pathology. This case presents a complicated identification of the pathology, due to a very unusual presentation of the lesion leading radiology, oncology and neurosurgery departments to believing that the lesion was a tumor with unspecified etiology. It was only surgical resection that allowed identifying the correct pathology of the brain lesion as a subacute stroke.

In general, primary brain tumor lesions are fairly rare. The incidences of metastatic lesions from esophageal adenocarcinoma were previously reported in the literature, estimating $2 \%$ of patients with esophageal carcinoma having brain metastasis. ${ }^{2}$ However, a more recent cohort study found $16 \%$ of patients with esophageal carcinoma having metastasis to the brain. ${ }^{3}$
Visual field defects after stroke are also well reported in the literature. The incidence of homonymous field defects was estimated at $8 \%$ in all patients with stroke with the majority (54\%) located in occipital lobes. ${ }^{4}$ The estimated lesions located in the lateral geniculate body (1\%) are published in the literature. ${ }^{4,5}$ Patients' impairments after stroke are significantly exacerbated by visual field defect. ${ }^{6}$

The most common clinical sign of infarctions in the posterior cerebral arteries distribution are visual field defect followed by neuropsychological deficits and higher order dysfunction including memory impairment as has been presented in this case. ${ }^{7}$ Acute confusional state and delirium are also common especially in the elderly population. ${ }^{7}$ Recent data suggested that confusion and delirium from a stroke in the right posterior cerebral artery distribution is significantly lower in comparison to the left or bilateral posterior artery distribution. ${ }^{8}$ Delirium was reported to remain for an appreciable poststroke period and was a marker of a poor prognosis. ${ }^{9}$ In this case, the patient is a well-educated and experienced engineer and the owner of an engineering company. The slow initiation and inferior performance, in light of his level of education, on the admission physical examination, and reported delirium state during the treatment before the admission to the rehabilitation floor, could be explained by the delirium and memory impairment after the posterior artery stroke. 


\section{Conclusion}

The importance of a detailed physical examination during admission cannot be overstated. The poststroke visual field defect can result in significant disability and reduction in quality of life. Patients with visual field cut can have a severely reduced quality of life and require additional neuropsychological and visual rehabilitation., ${ }^{4,6}$ Only a thorough physical examination during admission helped to challenge prior negative positron emission tomography scan data and led to the diagnosis of subacute stroke, and following appropriate treatment, secondary stroke prophylaxis and rehabilitation, instead of brain radiation and chemotherapy.

\section{Acknowledgments}

The authors thank Doctors Elina Chernyak and Marcia Scherer for their insightful review of our article and Doctors Mohamed Tejani, Nimish Mohile, Thomas Rodenhouse, and Carolyn Jones from the Strong Memorial Hospital, Rochester, NY, USA, for assistance in collection of data on the patient.

\section{Disclosure}

The authors report no conflicts of interest in this work.

\section{References}

1. Song DL, Zhong Y, Feng F, Li Y, Li MH. Homonymous quadrantanopsia as the first manifestation of invasive mole: a case report. $J$ Med Case Rep. 2012;6:117.

2. Weinberg JS, Suki D, Hanbali F, Cohen ZR, Lenzi R, Sawaya R. Metastasis of esophageal carcinoma to the brain. Cancer. 2003;98(9):1925-1933.

3. Smith RS, Miller RC. Incidence of brain metastasis in the patients with esophageal carcinoma. World J Gastroenterol. 2011;17(19): 2407-2410.

4. Luu S, Lee AW, Daly A, Chen CS. Visual field defects after stroke - a practical guide for GPs. Aust Fam Physician. 2010;39(6):499-503.

5. Tsuda H, Oi Y, Yoshioka M, Ishihara N, Yamada K. Homonymous hemianopia due to localized hemorrhage of the lateral geniculate body. Intern Med. 2006;45(21):1257-1258.

6. Gall C, Franke GH, Sabel BA. Vision-related quality of life in first stroke patients with homonymous visual field defects. Health Qual Life Outcomes. 2010;8:33.

7. Chao $\mathrm{AC}, \mathrm{Hu} \mathrm{HH}$. Acute confusional state with posterior cerebral artery infarction: a challenge in the diagnosis of acute stroke at bedside. Acta Neurol Taiwan. 2007;16(3):123-124.

8. Shih HT, Huang WS, Liu CH, et al. Confusion or delirium in patients with posterior cerebral arterial infarction. Acta Neurol Taiwan. 2007; 16(3):136-142.

9. McManus J, Pathansali R, Hassan H, et al. The course of delirium in acute stroke. Age Ageing. 2009;38(4):385-389.
Neuropsychiatric Disease and Treatment

\section{Publish your work in this journal}

Neuropsychiatric Disease and Treatment is an international, peerreviewed journal of clinical therapeutics and pharmacology focusing on concise rapid reporting of clinical or pre-clinical studies on a range of neuropsychiatric and neurological disorders. This journal is indexed on PubMed Central, the 'PsycINFO' database and CAS,

\section{Dovepress}

and is the official journal of The International Neuropsychiatric Association (INA). The manuscript management system is completely online and includes a very quick and fair peer-review system, which is all easy to use. Visit http://www.dovepress.com/testimonials.php to read real quotes from published authors. 